\title{
Erratum to: Electrical detection of dsDNA and polymerase chain reaction amplification
}

\author{
Eric Salm • Yi-Shao Liu • Daniel Marchwiany • \\ Dallas Morisette • Yiping He • Laila Razouk • \\ Arun K. Bhunia $\cdot$ Rashid Bashir
}

Published online: 9 August 2011

(C) Springer Science+Business Media, LLC 2011

Erratum to: Biomed Microdevices

DOI 10.1007/s10544-011-9567-x

The original version of this article unfortunately contained a mistake. An author on the paper, Laila Razouk was left out. The correct author list is shown above.

The online version of the original article can be found at http://dx.doi. org/10.1007/s10544-011-9567-x.

E. Salm $\cdot$ R. Bashir

Department of Bioengineering,

University of Illinois at Urbana-Champaign,

Urbana, IL 61801, USA

E. Salm $\cdot$ D. Marchwiany $\cdot$ R. Bashir

Micro and Nanotechnology Laboratory,

University of Illinois at Urbana-Champaign,

Urbana, IL 61801, USA

D. Marchwiany

Department of Molecular and Cellular Biology,

University of Illinois at Urbana-Champaign,

Urbana, IL 61801, USA

\section{Y.-S. Liu}

Taiwan Semiconductor Manufacturing Corporation,

Hsinchu, Taiwan 300, Republic of China

D. Morisette $\cdot$ L. Razouk

BioVitesse, Inc.,

West Lafayette, IN, USA
A. K. Bhunia

Department of Food Science, Purdue University,

West Lafayette, IN 47907, USA

Y. $\mathrm{He}$

USDA-ARS-ERRC,

Wyndmoor, PA 19038, USA

R. Bashir $(\varangle)$

Department of Electrical and Computer Engineering,

University of Illinois at Urbana-Champaign,

Illinois, IL 61801, USA

e-mail: rbashir@illinois.edu

Present Address:

D. Morisette

Birck Nanotechnology Center, Purdue University,

West Lafayette, IN 47907, USA 